\title{
Chapter
}

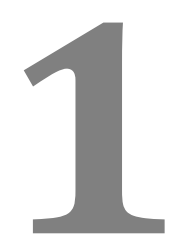

\section{POINT-OF-CARE TESTING IN LABORATORY MEDICINE}

\section{Joško Osredkar}

University Medical Centre Ljubljana, Clinical Institute of Clinical Chemistry and Biochemistry, Zaloška cesta 002, 1000 Ljubljana, Slovenia 


\section{Contents}

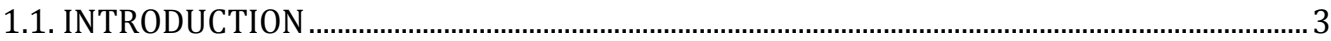

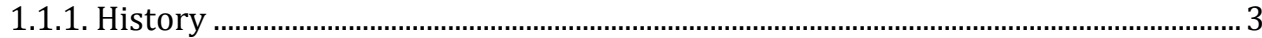

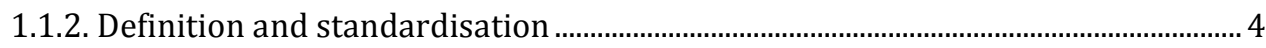

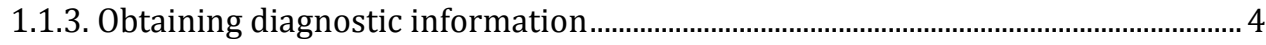

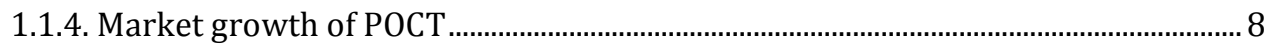

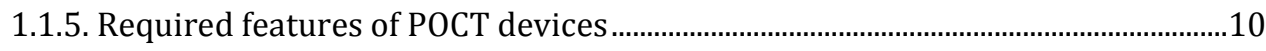

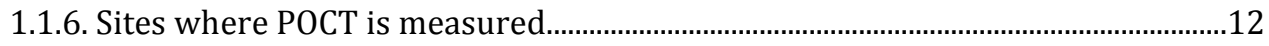

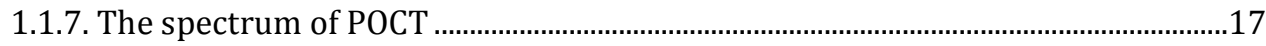

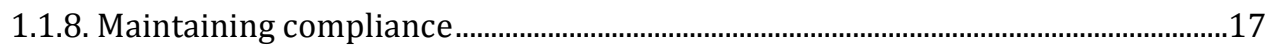

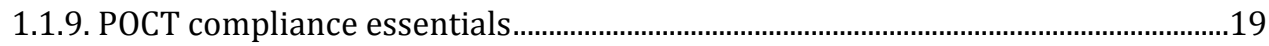

1.1.9.1. Device management .........................................................................................19

1.1.9.2. Quality control (QC) ..................................................................................19

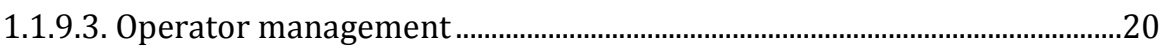

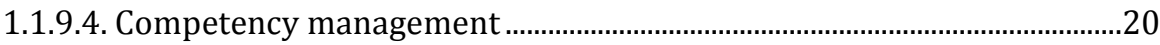

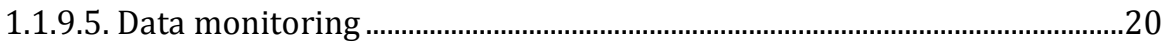

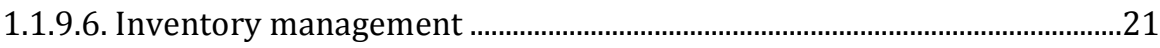

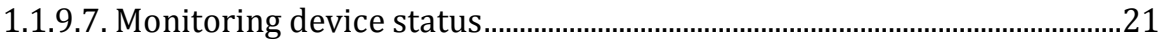

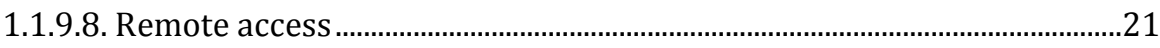

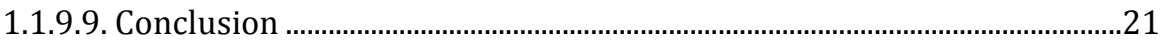

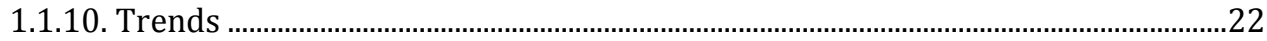

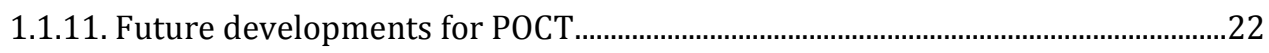

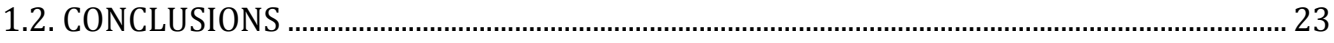

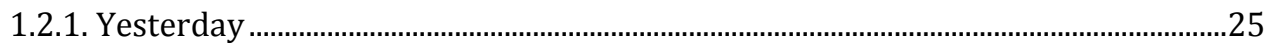

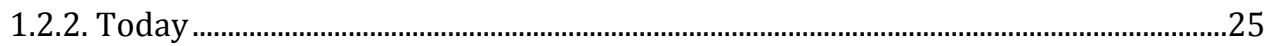

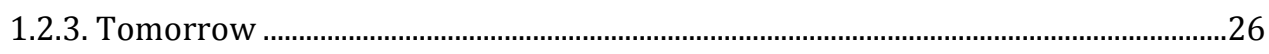

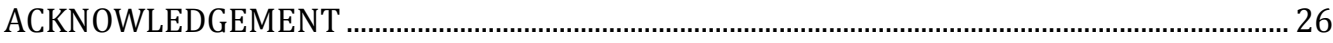

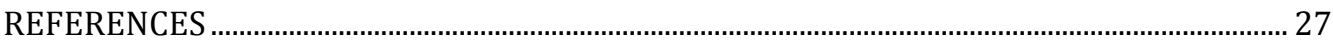




\subsection{INTRODUCTION}

\subsubsection{History}

The idea of rapid diagnostic tests on body fluids dates back to ancient history. One of the earliest records of a urine- based diagnostic test for pregnancy can be found in ancient Egypt. In this test (found in the "Berlin Papyrus"), a potentially pregnant woman could urinate on wheat and barley seeds over the course of several days. The result of this test: "If neither grows, she's not pregnant. If barley grows, it means a boy. If wheat grows, it means a girl" [1-3].

Hippocrates suggested that women who had missed their period should drink a solution of honey in water at bedtime. If they had abdominal distension and cramps, this would indicate the presence of a pregnancy [4].

Interest in urine as a rapid diagnostic medium continued through the Middle Ages. Physicians (such as the famous Avicenna) who claimed to diagnose different conditions from the colour of urine became known as "piss prophets" $[5,6]$. Another archaic example of a rapid diagnostic test was the practice of tasting a patient's urine to detect the presence of glycosuria, which indicates diabetes mellitus [7]. If it tasted sweet, it showed that the patient had diabetes mellitus (mellitus literally means 'sweet tasting' or 'honey'). Healers in 1500 $\mathrm{BC}$ noticed that ants were drawn to the urine of patients who had a mysterious emaciating disease. In the 1600 s, tasting the urine was the method to diagnose diabetes Sweet tasting urine=diabetes [8].

The first diagnostic test is thought to have been urine testing for diabetes. The earliest and most basic point-of-care (POC) test was dipstick urinalysis. The urine dipstick, developed in 1957, was the first true POC device. But it was not until the 1950's that the majority of rapid diagnostic methods gained real predictive value. The roots of lateral flow technology go back to the discovery of the antibody-antigen immunoassay reaction by Yallow and Burson in the $1960 \mathrm{~s}$ [3]. Together with the principles of thin layer and paper chromatography, it made a breakthrough possible. Clearly, the main application driving the early development of rapid-test technology was the human pregnancy test, which followed the continual historical interest in urine testing for medical diagnostic purposes. This testing application made great strides in the 1970s, as a result of improvements in antibody generation technologies and significant gains in understanding of the biology and detection of human chorionic gonadotropin (hCG) [3]. 


\subsubsection{Definition and standardisation}

POC testing (POCT) is defined as medical diagnostic testing performed outside the clinical laboratory and in close proximity to where the patient is receiving care [9]. POCT tests available at Capital Health include blood glucose, urine dipsticks, blood gases, chemistry, haematology, coagulation, cardiac markers, and pregnancy tests.

POCT is defined as "clinical laboratory testing conducted close to the site of patient care, typically by clinical personnel whose primary training is not in the clinical laboratory sciences or by the patients themselves (self-testing). POCT refers to any testing performed outside of the traditional, core or central laboratory" [10]. POCT is typically performed by non-laboratory personnel and the results are used for clinical decision making. POCT has a range of complexity and procedures that vary from manual methodologies to automated analysers.

POCT devices are often 'hand held' or may be small portable analysers. POCT is generally more expensive than lab testing but is appropriate and cost effective in some clinical settings because testing is performed near the patient and informs immediate decisions for the clinical management of the patient $[5,6]$.

According to this definition, there are many synonyms for this form of testing as presented in Table 1 [10].

Table 1. Synonyms for POCT

\begin{tabular}{cc}
\hline POCT & Home testing \\
\hline Ancillary testing & Self-management \\
\hline Satellite testing & Patient self-management \\
\hline Bedside testing & Remote testing \\
\hline Near patient testing & Physician's office laboratories \\
\hline
\end{tabular}

\subsubsection{Obtaining diagnostic information}

Process in obtaining results comprises pre-analytical, analytical, and postanalytical phase. Pre-analytical phase includes the phase from ordering the test till the moment of analyse on instrument. Analytical phase involves the preparation of the sample, analysis and quality control (QC) measures. Postanalytical phase begins with further validation of the results with interpretation [11].

Detailed activities in certain phases are listed in Table 2. 
Table 2. Activities involved in different phases of analytical procedure

Identifying the correct test
Determining proper conditions
(fasting, time of the day, resting, posture, taking of drugs
Request form with clinical data
Taking the sample
Labelling of the tubes
Transport of the sample
Areparation of the sample for instrument
AC requirements
Comparing the result against reference ranges
Proceeding the result to the requesting personnel
Interpreting the results (in the case of abnormal results)
Interpretive comments

POCT is an established part of clinical practice, in many instances offering the rapid and convenient provision of test results that can be of benefit to the patient [12]. It is, however, a significant risk management issue for the trust. Results obtained by non-specialist staff carry as much weight in patient management as results from sophisticated equipment in the care of professional laboratory staff. Incorrect results have the same health and legal implications, whatever their origin.

There is some ambiguity concerning the term POCT and its predecessors, bedside testing, near patient testing, and less frequently, ancillary or decentralized testing. All were derived from the proximity of the laboratory test to the patient or central laboratory, but this distinction is relative and imprecise. POCT is often regarded as tests performed outside of a central laboratory, but this definition also is unsatisfactory because limited-service satellite laboratories staffed by laboratory personnel are considered clinical laboratories (or sometimes blood gas laboratories) but not POCT services, at least for accreditation and regulatory standards. Their location near the patient does not influence the accreditation standards they must meet.

POCT is defined as wany test that is performed at the time at which the test result enables a decision to be made and an action taken that leads to an improved health outcome«.

We need to break down the turnaround time (TAT), to see where the time was being spent. We broke down the TAT into seven steps, as presented in Figure 1 [13]: 


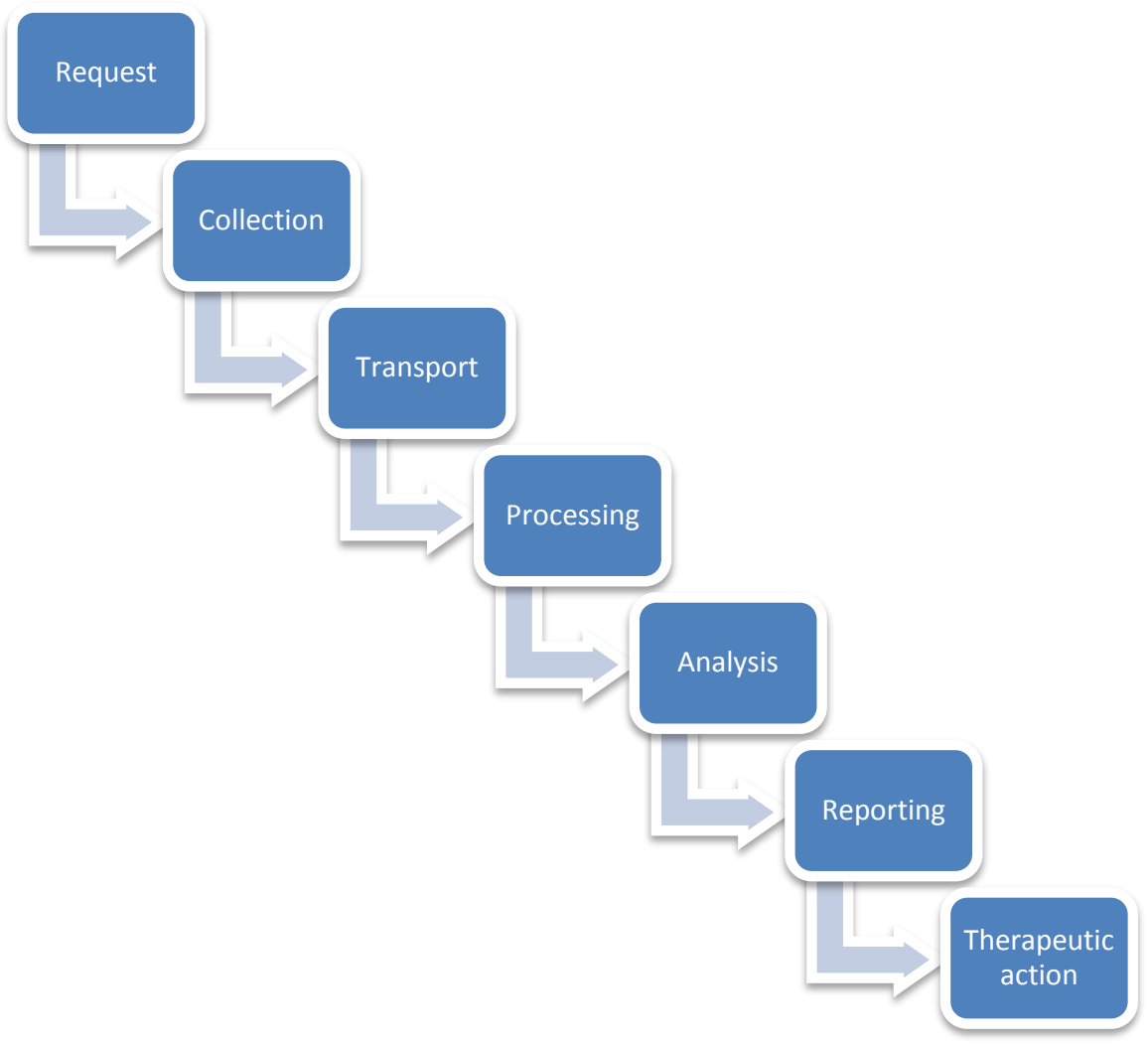

Figure 1. Step involved from doctor order of specific test to result from the laboratory

This was the therapeutic turnaround time (T-TAT): from the time of the request to the time when therapeutic or patient management action was taken. We then look at the time for each step. In most cases, we can find the time for the verbal request (which would be entered into the computer after analysis), collection, transport (with the OR close to the laboratory), and reporting. The remainder of the TAT is spent in spinning the specimen down and performing the analysis. We know we need to look at all the steps of the T-TAT: those occurring outside the laboratory as well as those inside the laboratory to significantly reduce the TAT [14].

For regulatory purposes, satellite laboratories are generally considered extensions of the central laboratory service rather than a separate classification such as POCT. Therefore, the location at which a laboratory test is performed does not classify it one way or the other. It is even conceivable, given the following considerations, that POCT could be performed in a central laboratory. 
Yet another criterion for defining POCT - and possibly the most satisfactory definition from a regulatory perspective - is who performs the test. If laboratory personnel perform a test, then this test typically falls under the laboratory license, certificate, and accreditation, even if it is performed outside of the physical laboratory space and regardless of whether the test is waived or non-waived. On the other hand, waived or non-waived laboratory tests performed by non-laboratory personnel are nearly always subject to a different set of regulatory and accreditation standards, and these can be grouped neatly under the POCT umbrella.

Therefore, POCT somewhat misleadingly suggests a location where the test is performed, but in fact the regulatory standards are primarily determined by who performs it. In practice it is highly unusual for non-laboratory personnel to perform any tests within the clinical laboratory, but the converse is relatively common. Clinical laboratory personnel often perform laboratory tests outside the central laboratory (e.g., Streptococcus A screening in an emergency department, sweat tests in a paediatric ward, urine drug screens in the human resources department, international normalized ratio in a coagulation clinic). Tests like these fall under the clinical laboratory improvement amendments (CLIA) certificate and, in states that issue them, the laboratory license. Consequently, POCT typically refers to waived or nonwaived laboratory tests performed at remote locations by non-laboratory personnel.

Guidelines and/or standards for POCT have been issued by a number of organisations to assure patient safety and the quality of:

- Following patient deaths, The Department of Health issued Hazard Notice (1989) to all Clinical providers of POCT, recommending Pathology involvement in managing POCT.

- The Joint Working Group on Quality Assurance (JWGQA) has published guidelines, updated in January 1999, for the implementation of POCT, covering procurement, QC and assurance, health and safety, risk management, user training, equipment maintenance and clinical liaison: Near to patient or POCT guidelines issued by JWGQA to assist health service managers and staff in the procurement, installation use and monitoring of devices suitable for performing tests on blood, urine and stools (2000).

- Clinical Pathology Accreditation (UK) Ltd, the national quality standards body for Pathology Laboratories, which includes new standards for POCT (both ISO 15189:2012 and ISO 22870:2006 apply to POCT).

- The Medicines and Healthcare products Regulatory Agency (formerly The Medical Devices Agency), following professional consultation, has issued guidance on the management of POCT: Management and use of in vitro diagnostic (IVD) POCT devices (December 2013). 
The POCT policy has been designed to ensure that POCT at Homerton University Hospital Foundation Trust (HUHFT) complies with all such advice and guidance.

\subsubsection{Market growth of POCT}

POCT is one of the fastest growing aspects of clinical laboratory testing. It is estimated to be increasing by at least $10-12 \%$ per year, with some areas increasing $30 \%$ per year. In contrast, central laboratory testing has grown 6-7 \% per year. Various reports are available to document the growth of IVD markets including various categories such as POCT. While the numbers vary between reports, the total IVD market was believed to be worth 51 billion USD in 2011, of which approximately 15 billion USD was POCT. The latter is projected to show compound annual growth of $4 \%$ to reach 18 billion USD by 2016 [14-18].

This examination of POCT focuses on the POC segments in important worldwide markets, such as the U.S., Japan, Europe, Asia and Rest-of-the-World (ROW). Of the total POCT market in 2011, $55 \%$ of it was in the US, $30 \%$ in Europe and $12 \%$ in Asia. An extensive review of POCT in this report includes the market for clinical equipment and supplies, as well as the market for screening reagents and instruments for the analysis of individual components in blood, serum, urine and other body fluids. This report defines the dollar volume of sales, for both worldwide and U.S. markets, and it analyses the factors that influence the size and the growth of the individual market segments. Most of the companies known to be developing instruments and reagents for the clinical POCT market are examined in this study. Each company is discussed in depth with a section on the company history, the product line, a business and marketing analysis and a subjective commentary on the position of the company in its market.

TriMark estimates that the professional global IVD testing market was valued at approximately 62 billion USD in 2015 and that the professional POCT market sub-segment was valued at 12.91 billion USD in 2015, or the equivalent of $19.4 \%$ of the global IVD market [19,20]. Numbers expressed as \% are graphically presented in Figure 2. 


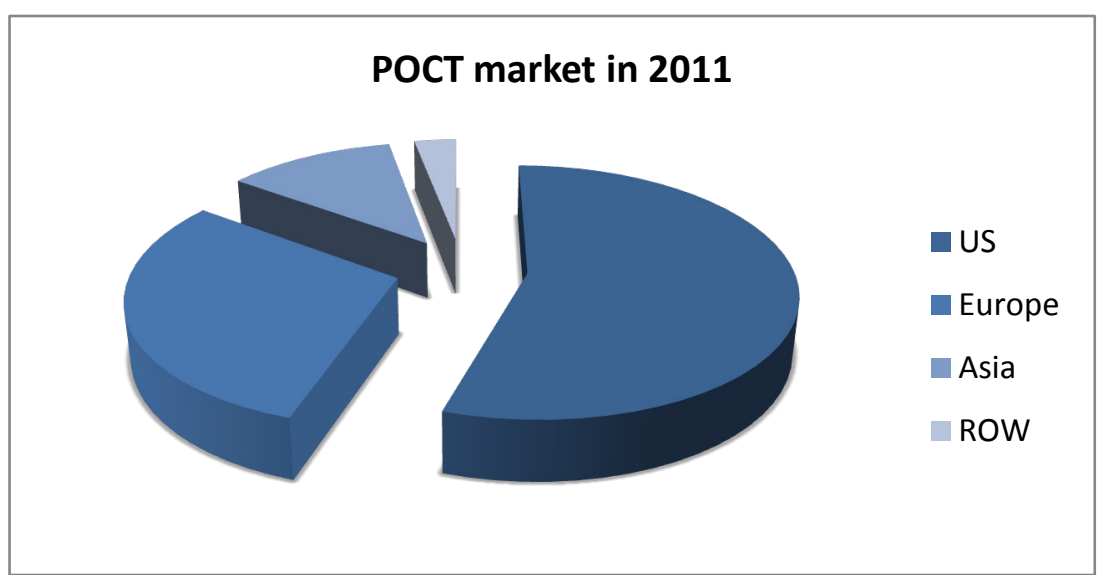

Figure 2. IVD market share in 2011

The report provides a summary of the global POCT market divided into individual major market sub-segments, with market values for 2015 and market projections to 2018.

North America held the largest market share of over $43 \%$ in 2015 due to the presence of high disease prevalence levels in the region coupled with favourable government regulations and initiatives pertaining to the development of healthcare infrastructure [20]. Moreover, the presence of high awareness levels amongst the patients and the physicians pertaining to early disease diagnosis is supportive for the largest share of the region in the industrial revenue.

The U.S. is recognized as the largest single country market for IVD and POCT products, but Europe represents the largest regional market for both of these. Germany is widely recognized as the largest single market for both IVD and POCT products within Europe [19,21].

The Asia Pacific area is expected to be the most attractive regional market space, owing to the high presence of unmet medical needs and the constantly improving healthcare infrastructure, increasing medical awareness, and the rise in per capita income levels in developing economies of including India and China [20].

Asia (which includes Japan, China and India for the purposes of the report) represents a major market, but there are significant differences in growth rates for each of the individual countries. TriMark estimated that the Asian POCT market would exhibit an annual growth of $12.2 \%$ between 2015 and 2018. Although the Japanese POCT market has slowed recently, in contrast, the emerging markets of India and China are exhibiting higher annual growth. The report also provides a summary of the Asian POCT market segmented by individual major market sub-segment with market values for 2015 and market projections until $2018[19,22]$. 


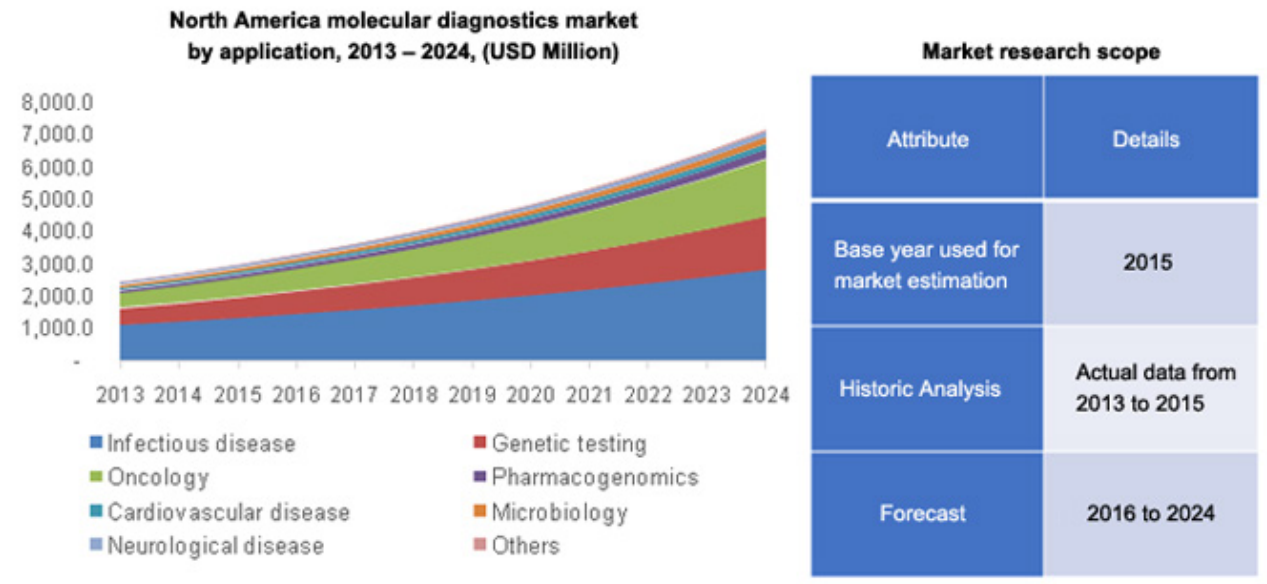

Figure 3. U.S. POC diagnostics market for the period from 2013 to 2024 by product

Blood glucose testing POC meters accounted for the largest share of over $40 \%$ in 2015 due to the sheer volume of these tests marketed on account of their high usage.

The POC cardiac marker testing segment is expected to witness relatively faster annual growth over the forecast period. The primary factor driving R\&D in the segment is the fact that deaths related to cardiovascular disease have increased by over $40 \%$ over the past two decades [20].

\subsubsection{Required features of POCT devices}

One of the most important considerations for product developers who are thinking about the challenges for POC solutions is developing a strong collaboration between industry, clinicians, laboratorians and patients. Including all the stakeholders in development of new product requirements ease of use, access, test menu, instrument features and performance, connectivity - can ensure that the changing ecosystem of POC is effectively incorporated. Product developers start with the needs of their users which to some extent depend on the clinical setting. The laboratory professional is not the typical user of POCT, but a group of people who don't have formal training to use it: clinicians, nurses, etc. Taking into account, then, better POCT devices are those that fulfil basic requirements:

- simple to use,

- reagents and consumables are robust in storage and usage,

- results should be concordant with an established laboratory method. 
The device and the associated reagents and consumables are safe to use $[14,23,24]$.

Here are several additional recommendations to manufacturers:

- minimize the number of parts and steps to obtain a result,

- avoid a confusing product design, such as colour-coded swabs, which can affect accuracy,

- eliminate manual specimen application with a metered dose

- make the results easy to interpret,

- make tests that withstand use, like cleaning with bleach or dropping.

The most robust devices are those that enable the majority of the steps involved in performing an analysis.

Operator considerations are not the only criterion for choosing an appropriate POCT device. Others are analytical performance (accuracy, precision and comparison to laboratory values), reagent requirements and cost.

POCT methods are to a large extent based on those methods used in the laboratory. Devices are handheld, handheld with meter reading and benchtop. We can measure single analyte or combinations as listed in Table 3. Handheld devices are mainly for single analyte, while other two variants for more than one.

A handheld device which is used in the largest number is dipstick for qualitative urine testing. This device is very simple to use but operator should pay attention to:

- the need to cover the whole pad with the sample and,

- the need to time the period between placing the sample on the pad and comparing the resulting colour to colour card [11].

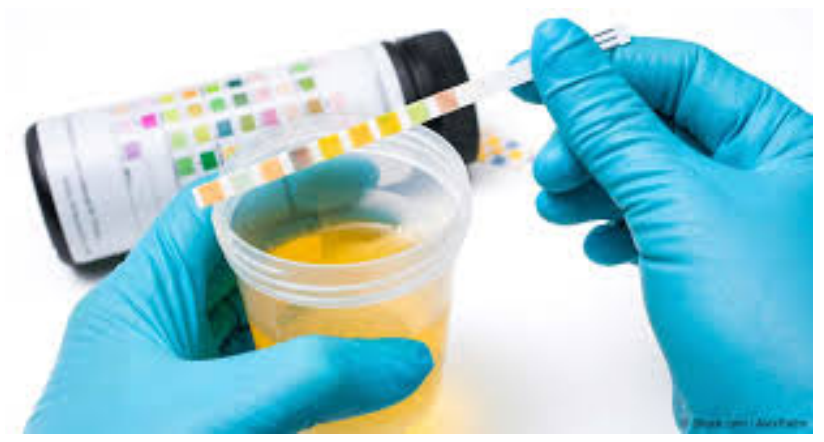

Figure 4. Dipstick for urine testing 
POC test can be any of the following (Table 3):

Table 3. POCT for single and multiple analytes testing

\begin{tabular}{cc}
\hline Pregnancy \& Fertility Test kit & Cardiac Marker Kit \\
Blood Glucose Testing kit & Blood Gas/Electrolyte Test Kit \\
Cholesterol Test kit & Saliva Test Kit \\
Coagulation & Hematology Test Kit \\
Drug Abuse Test Kit & Food Pathogen Test Kit \\
\hline Infectious disease Test Kit & Allergy Test Kit \\
\hline Urine Test Kit & Faecal Occult Test Kit \\
\hline Oncology Test Kit & Miscellaneous Testing \\
\hline
\end{tabular}

The POC market is growing due to advancement in technology and the demand for quick test results. Also the increase in the prevalence of infectious disease and lifestyle diseases like cardiac disease and diabetes, along with the aging population is increasing the demand for POC diagnostics. Awareness among people of the easy and convenient way to get faster test results is increasing the demand physicians also prefer POC their quick decision making when prescribing a disease-specific drug, which was previously unlikely when doctor started symptomatic treatment as the test procedure could take a long time. There is a tremendous demand for POC in the Americas region, and the increase in awareness in the Asia-Pacific region will lead to tremendous growth in the future as the highest population countries in Asia will drive it tremendously. The traditional method of clinical testing required skilled labour while POC required minimal training, thus reducing the costs of training and the time consumed to perform the test. The major restraint for POC test market is the unwillingness to change traditional treatment practice.

\subsubsection{Sites where POCT is measured}

The two most important areas where such tests are measured for immediate results in a POC setting are hospital emergency rooms and critical-care clinics [19]. The third place where these tests are frequently measured in what is characterized as a near-patient setting is in physicians' office labs (POLs). Other testing areas of interest for these analytes are satellite labs, critical-care units, neonatal intensive-care units (NICUs), intensive-care units (ICUs) and home testing locations. Home testing is not directly covered in this report except in cases when the products and companies in this market segment are also actively used in the hospital and physician's POC settings.

In every setting (hospital, institution or medical clinic point-of-care testing), a written Point-of-Care Program/Policy is important since point-of-care testing 
tends to expand rapidly and gets out of control unless guidelines or policies are in place [25]:

A written POC program / policy is important since POCT tends to expand rapidly and can get out of control unless guidelines or policies are in place.

The "Program / Policy" should clearly define:

1. Who is responsible for each part of the program naming the key people? For example:

a. Laboratory POC Coordinator: keep data base of testing personnel, coordinate the training of new personnel, choose testing methods, monitor QC and proficiency programs, provide ongoing coaching to testing personnel in response to daily monitoring, consulting on technical issues, and analysemeter/troubleshooting.

b. Nurse Manager: enforce policies, schedule new employee training, take disciplinary action, if necessary, and schedule annual POC competency evaluation of staff.

c. Education dept. (if it exists): new employee training and annual certification of testing personnel, support committee with agenda and minutes of meetings. Preferably training is done by those reviewing daily results and quality monitoring.

d. Laboratory staff: new employee training, aid in the annual certification of testing personnel, download and/or review QC data, verify equipment function and maintenance.

2. Where the testing will be performed and who will perform it?

3. For what purpose each type of POCT will be used, i.e., screening, diagnosis, treatment?

4. Who will chose the methodologies used, i.e., lab, Point of care Coordinator (POCC)?

5. What method validation procedures will be performed prior to implementation and who will perform the validation?

6. Reporting procedures.

7. Staff training, continued competency programs and feedback/communication with the end users.

8. Quality assurance monitoring protocols including QC protocols.

9. Proficiency testing program.

10. Obtain and maintain the appropriate licensure and compliance with regulations.

11. Protocol for requesting new/additional services.

12. Operational budget [13]. 
Early POCT methods were mostly manual, with minimal or no QC and limited data management capabilities [26].

Modern POCT devices are greatly improved, but capturing the data required to document compliance remains a labour-intensive process. In addition, despite the rapid growth of POCT methods and use, POCT operators often have a limited understanding of the regulatory and accreditation requirements for licensure, training, procedures and documentation. Consequently, nurses and other providers often see POCT coordinators as police who indiscriminately enforce regulations that seem onerous at best and detrimental to patient care at worst. This is an unfamiliar and uncomfortable role for laboratory medicine professionals, who are highly trained to promote quality patient care and the efficient use of resources. In this brief review, we will discuss some POCT-related regulatory issues in the hospital environment, and potential ways to satisfy those requirements [27].

Much of the research and development is aimed at bringing testing to underserved populations and developing nations. Even in patients who are at high risk, testing is limited by several factors: expense and location. Most testing takes place in centralized or regional laboratories. The WHO has developed guidelines for the development of diagnostics for low-resource settings - ASSURED (Figure 5) [18]:

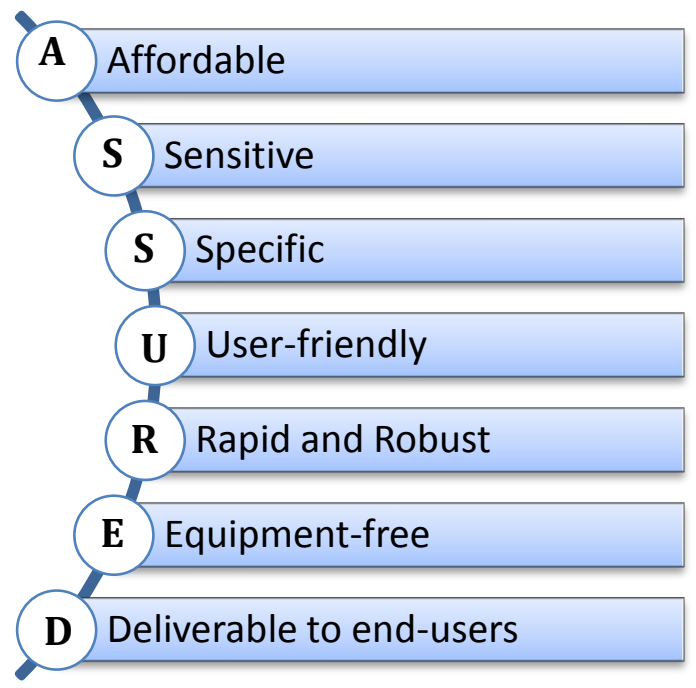

Figure5. WHO developed guidelines

Technical advances are making POC testing more accurate, more robust, cheaper and easier. Changes in the clinical environment are prioritising shorter hospital stays and quicker patient turnaround and new therapies that 
require rapid laboratory results are appearing in hospitals and outpatient clinics. Heavy promotion by industry due to favourable profit margins is also increasing the shift of care to the home setting. Not just blood glucose testing, but self-monitoring of anticoagulation can be used in conjunction with telemedicine to care for patients with chronic conditions. "With the development of miniaturized devices and wireless communication, the ways in which doctors can care for patients will change dramatically and the role the patients take in their own healthcare will increase. Healthcare will become more personalized through tailoring interventions to individual patients. The next decade will bring a new realm of precision and efficiency to the way information is transmitted and interpreted, and thus the way medicine is practiced. In the future, clinicians may be able to improve the regulation of diet in infants with inborn metabolism issues through bedside monitoring. Currently, the management of such diseases requires complex testing in a hospital setting. However, researchers are developing a chemical sensor using a small sample of blood from a finger stick, which changes colour in response to metabolic irregularities. When such abnormalities are found, the diet of the infant can be adjusted immediately to prevent adverse effects such as mental retardation.

TriMark estimates that future growth will stem from emerging applications like genotyping for identifying drug-resistant strains; bioterrorism; testing applications within infectious diseases like Influenza and HIV; and disease diagnostics and prognostic assays for disease applications such asepsis, cardiovascular disease (CHF) and coagulation testing. The industry consolidation is significant, as larger players like Alere want to move into faster-growing markets to expand their product offerings and/or geographical reach. Larger, established diagnostic players like Roche are eager to build out and extend their molecular diagnostic franchises to cover POC technology and are willing to pay premium prices for good technology [19].

Detailed charts with sales forecasts and marketshare data are included. For more information, visit:

https://www.trimarkpublications.com/products/Point-of-Care-DiagnosticTesting-World-Markets.html.

Improvements in technology and the resultant introduction of cost effective and high quality medical solutions aimed at achieving lab automation and miniaturization are anticipated to be an important driver in the global POC diagnostics. Moreover, increasing levels of patient awareness are expected to prove an imperative for the growth on account of the fact that patients, especially those suffering from long term diseases such as diabetes, are willingly participating in the shift towards the implementation of POC diagnostic practices [20]. 
Additionally, healthcare practitioners have been observed to be increasingly more inclined towards using POC diagnostic medical devices and consumables, thereby resulting in a growing adoption and demand for POCT solutions.

The development of information technology pertaining to healthcare systems plays an inevitable role in the development of the POC diagnostics market. The introduction of healthcare information systems such as electronic health records (EHR) has significantly increased the penetration rates of these tests as a combination of the afore mentioned, enabling considerable automation of diagnostics capabilities of any medical facility.

The growing prevalence of the target diseases coupled with the rise in the geriatric population, which is more prone to disease and require home care and monitoring, will also impact the product demand considerably over the forecast period [28].

Beginning in the 1980s, POCT departed from conventional clinical laboratory medicine by decentralizing laboratory services. Deploying POCT devices created challenges for laboratory management, especially in ensuring the proper use of these devices. Today, POC coordinators use a variety of processes to maintain control of multiple devices and to monitor the regulatory compliance of many operators at locations scattered across healthcare enterprises. The confidence of all three parties (doctor, operator, patient) in the results depends on robustness of the method and competence of the operator. Operator should demonstrate the competence for performing tests, and therefore there must be an effective training program. The key elements are listed in Table 4:

Table 4. Key elements of a training program

Understanding the concept of text (pathophysiological, clinical requirements, action taken after performing the test, nature of the test)

Patient preparation (biological variability, lifestyle, therapy, diurnal variation)

Sample requirement and specimen collection

Preparation of instrument

Performance of test

QC

Documentation

Reporting the result

Interpretation of the result

Health and safety issues (specimen collection, disposal of sample, cleaning of the instrument)

Competence should be maintained with continuing education. Audit must include the review of analytical performance (QC, quality assurance data) [11]. 


\subsubsection{The spectrum of POCT}

There is some ambiguity concerning the term POCT and its predecessors, bedside testing, near patient testing and, less frequently, ancillary or decentralized testing. All were derived from the proximity of the laboratory test to the patient or central laboratory, but this distinction is relative and imprecise. POCT is often regarded as covering tests performed outside a central laboratory, but this definition also is unsatisfactory since limitedservice satellite laboratories staffed by laboratory personnel are considered clinical laboratories (or sometimes blood gas laboratories) but not POCT services, at least for accreditation and regulatory purposes. Their location near the patient does not influence the accreditation standards they need to meet.

For regulatory purposes, satellite laboratories are generally considered extensions of the central laboratory service, rather than a separate classification such as POCT. Therefore, the location where the laboratory test is performed does not classify it one way or another. It is even conceivable, given the following considerations, that POCT could be performed in a central laboratory.

\subsubsection{Maintaining compliance}

Regulatory oversight of POCT differs in several respects from that of other clinical laboratory services, and maintaining compliance with the agencies involved in POCT oversight can be a daunting task. Federal regulation of POCT is minimal and the only requirement for most tests in this category is that they must be performed according to the manufacturer's instructions. However, states and accrediting agencies often impose additional requirements on POCT that healthcare facilities need to deal with. These requirements primarily focus on operator competency and verification that the procedures specified by the POCT manufacturer are strictly followed.

Numerous POCT related publications are available to help organizations implement POCT. A list is not exhaustive:

Clinical and Laboratory Standards Institute. POCT02-A: Implementation Guide of POCT01 for Health Care Providers; Approved Guideline, 2008

National pathology accreditation advisory council. Guidelines for POCT (1 ${ }^{\text {st }}$ Ed. 2015)

AACC - Monitoring POCT Compliance. FEB.1.2016

RiliBÄK (Richtlinien der Bundesärztekammer).

The term 'RiliBÄK' Guidelines ("Rili") of the German Federal Medical Council (BÄK)

International Federation of Biomedical Laboratory Science (IFBLS) -

The Guidelines for POCT and the EPBS Policy Statement. October 2009

The European Association for Professions in Biomedical Science (EPBS) -

The Guidelines for POCT and the EPBS Policy Statement. Zagreb 2015

Association of Clinical Biochemists in Ireland - ACBI. Guidelines for Safe and Effective Management and Use of POCT in Primary and Community Care 
States vary in the degrees to which they regulate POCT. For example, Florida a state that licenses clinical laboratories and the technical personnel employed by them - has minimal regulations for waived tests but very strict requirements for non-waived laboratory tests performed by personnel without a clinical laboratory technician or technologist license. The Florida Administrative Code (FAC) specifies the qualifications necessary for nonlaboratory personnel performing non-waived tests, a category of laboratory testing the FAC refers to as alternate site testing. To perform alternate site laboratory tests in Florida, the employee must be a licensed healthcare professional under any one of several categories, including physician, dentist, physician's assistant, nurse (registered nurse (RN), licensed practical nurse (LPN) or advanced registered nurse practitioner (ARNP)), respiratory therapist, etc. Thus, the principal regulatory requirement focuses on personnel qualifications, not the proximity of the test to the patient or the laboratory.

However, the FAC imposes additional requirements that disqualify the vast majority of non-waived laboratory tests from being performed by nonlaboratory personnel regardless of their qualifications. The tests must use whole blood and must not require specimen manipulation, such as manual dilution or centrifugation. In addition, the instrument must be self-calibrating and equipped with failsafe mechanisms that prevent the results being reported in the case of calibration or QC failure. Therefore, even though non-waived testing is allowed at alternate sites, Florida law strictly limits the variety of non-waived tests that can be deployed in a POCT environment.

As with clinical laboratories, compliance with state and federal requirements for POCT are ordinarily met through accreditation by organizations with deemed authority, such as the College of American Pathologists (CAP) Laboratory Accreditation Program or the Joint Commission. Although the accreditation standards recognized by these organizations meet the centre for medicare and medicaid services (CMS) and state requirements, they are not identical in all respects. Therefore, some hospitals may choose to have their clinical laboratories accredited by one organization, and their POCT program accredited by another [29].

CMS does not require all laboratory services to be accredited by the same organization as long as each clinical laboratory improvement amendments (CLIA) certificate is covered by a deemed authority, and in some respects the accreditation standards of one organization may be easier to satisfy in a particular setting than those of another. As a result, it is not unusual for a hospital to have its clinical laboratory services accredited by, for example, the CAP, while their POCT program is accredited by the Joint Commission.

In general, POCT regulatory requirements focus on two areas: the training and competency of the personnel doing the testing, and verification of strict adherence to the manufacturer-specified procedure for each test. The latter focus is particularly important because waived or moderately complex 
laboratory methods, both of which can be performed by non-laboratory personnel under certain circumstances, become highly complex if used in a way that deviates from the FDA-approved manufacturer's protocol. Highly complex laboratory tests, by federal law, can only be performed by personnel meeting the qualifications specified in CLIA Subpart $M$, and additional educational and licensure requirements may be imposed by some states.

Since the high complexity essentially eliminates a laboratory test from consideration for POCT, it is critically important that supervision of POCT in a healthcare institution includes verification that testing procedures do not deviate from the manufacturer's instructions.

POCT device manufacturers have responded to the challenge of monitoring the use of these instruments by designing features such as access control and electronic communication with a laboratory information system (LIS) or other network system. This communication allows the LIS to download QC and patient results. However, POCT coordinators still need a dedicated resource for managing their POCT programs. The use of connectivity via a data management system can significantly improve efficiency when managing various aspects of compliance. With the adoption of POCT1-A communication protocols, data systems evolved from vendor-specific to vendor-neutral platforms. Although there may be some functional limitations for specific devices, vendor-neutral platforms offer POCT coordinators the flexibility to connect devices from multiple manufacturers, providing better support for the compliance elements of the program.

\subsubsection{POCT compliance essentials}

\subsubsection{Device management}

Device management is a key as central laboratory tests continue their migration to POCT platforms. POCT devices can be set up and configured remotely from a single central location with software updates manually or automatically downloaded to the devices. In addition, the data management system serves as a repository for testing locations, instrument serial numbers, instrument service history and software versions. The data management system also tracks the status of the connected devices so that communication and connectivity issues can be addressed promptly.

\subsubsection{Quality control (QC)}

QC is required for all waived and non-waived tests. The QC limits and frequency intervals can be configured in the device or managed remotely with the data management system. This prevents an operator from using the instrument once the QC interval has been exceeded or if the result is not within acceptable limits. The QC results for each device and operator can also be 
reviewed and evaluated by laboratory personnel, which is a requirement for most laboratory accreditation programs. The data system also captures and stores comments describing corrective action for unacceptable QC results. Some data management systems allow QC import into other software programs for peer comparison, as well as the capture of manual QC results for tests such as faecal occult blood.

\subsubsection{Operator management}

Operator management refers to controlling access to POCT devices and tracking the authorization of all operators, with alerts when certifications have expired (see 1.1.9.4. Competency management). Access to a POCT device can be authorized via operator list downloads when the instrument queries the data management system to determine whether an operator is currently certified. If an operator with expired certification attempts to use the POCT device, he or she will be locked out, preventing use. Some data management systems notify operators when they are approaching the expiration date of their access to a device.

\subsubsection{Competency management}

Data managements systems enable POCT coordinators to track the dates on which operator competencies were completed for original certification, recertification, QC performance, patient results and reporting. This increases efficiency, especially when paired with other learning management systems (LMS) such as Healthstream. Although both systems currently require some manual input and maintenance of data, the ability to interface the data management system with the LMS may be on the horizon. Currently, data management systems include the ability to generate reports that show initial training, 6 month, and yearly competencies, all of which are required elements under waived and non-waived testing standards. Many systems also offer the automatic recertification of operators.

\subsubsection{Data monitoring}

In order to comply with the accreditation standards, POCT coordinators monitor data from activities such as correlation testing, linearity and analytical measurement range verification, proficiency testing, calibration and patient identification. Data systems can automatically capture this data and document it for review. This data also can be entered by hand from manual tests (e.g. faecal occult blood, dipstick urine, pregnancy tests), although compliance with these POCT standards that does not involve interfaced instruments is difficult to verify. While instrument platforms exist for each of the previously mentioned tests, they are more commonly performed manually. 


\subsubsection{Inventory management}

Data systems are also essential for managing consumables for POCT devices. These tools include reports showing usage and device workload that laboratorians can use to establish the frequency and size of supply orders, potentially reducing costs by eliminating the waste of expired reagents and controls. Reagent and control lot numbers, as well as established QC ranges, can be entered into the data system and uploaded to the POCT devices. In addition, alarms can be set to alert the POCT coordinator when new lots are in use that may require validation. Many POCT devices include barcode scanning capabilities that allow reagents and controls to be scanned by operators to verify the current lot number and prevent the use of expired or unvalidated reagents. The current lot numbers may reside in the data management system.

\subsubsection{Monitoring device status}

Remote monitoring enables a POCT coordinator to determine the status of any connected devices. Inoperable devices can be identified immediately and either removed from service or repaired. For example, many POCT devices have a data buffer that, when exceeded, prevents the device from being used until the buffer is cleared. This type of error can be detected by the data management system and dealt with promptly by testing or supervisory personnel. By configuring alerts, the data system may also give coordinators the ability to investigate and resolve issues before they become critical.

\subsubsection{Remote access}

Remote access enables POCT data management from a computer anywhere within or outside of the organization, based on how the system is configured. With the adoption of mobile devices such as tablets and smartphones, webbased data management applications can be accessed from virtually anywhere to exchange information and manage systems, including the ability to send remote commands to the devices in some cases.

\subsubsection{Conclusion}

Regulatory oversight of POCT focuses primarily on ensuring the proper training and competency of the personnel performing these tests, as well as verifying that the tests are being conducted according to manufacturer instructions. Supervision of a POCT program requires attention to these and other aspects of laboratory tests performed by non-laboratory personnel. Connectivity via data management platforms has provided an elegant solution to the challenge of managing these regulatory and compliance aspects of a large POCT program.

With the widespread implementation of wireless networks, and the built-in WiFi capabilities of most modern analytical devices, data management systems for POCT will eventually support a seamless network of POCT devices 
deployed throughout a healthcare facility, perhaps fundamentally changing our notion of a clinical laboratory is.

As more and more laboratory services move outside our traditional workspace, laboratory medicine professionals face increasing responsibilities to ensure the quality and integrity of laboratory services throughout the entire facility. We have outlined just a few of the regulatory and accreditation issues that accompany the supervision of a POCT program [29,30].

Considerable advances in POCT devices stem from innovations in cellphone (CP)-based technologies, paper-based assays (PBAs), lab-on-a-chip (LOC) platforms, novel assay formats, and strategies for long-term reagent storage. Various commercial CP platforms have emerged to provide cost-effective mobile healthcare and personalized medicine. Such assay formats, as well as low-cost PBAs and LOC-based assays, are paving the way towards robust, automated, simplified, and cost-effective POCT [32]. Strategies have also been devised to stabilize reagent storage and usage at ambient temperature.

Nevertheless, the successful commercialization and widespread implementation of such clinically viable technologies remain subject to several challenges and pending issues.

\subsubsection{Trends}

Recent advances in emerging technologies (i.e., CP-based technologies, PBAs, and LOC platforms) are paving the way for next-generation POCT.

Advances in novel assay formats and strategies for long-term reagent storage are the prerequisites for emerging POCT technologies.

Current and future analytes for POCT comprise small-molecule metabolites, proteins, cardiac biomarkers and cells.

One emerging future trend is centred on miniaturized, fully automated, and network-enabled CP-based POCT technologies integrated with PBAs and/or LOC platforms.

\subsubsection{Future developments for POCT}

Given the rate of technological advancement and the potential benefits to efficiency and quality of care offered by POCT, it seems likely that the prevalence of POCT in healthcare will continue to grow in the future. Government initiatives, along with a high incidence of time-sensitive medical conditions, already provide strong incentives for the expansion of POCT in hospitals and emergency departments. In the surrounding community, financial incentives and trends toward increased patient involvement in their own care (empowerment of the patient) will likely continue to drive the expansion of POCT outside hospital centres. When POCT enters the community, issues concerning management and oversight, training, quality assurance, and documentation are all significantly amplified [32]. In the UK 
there are various pressures, not least from the government, to move pathology testing closer to the patient (i.e. general practices, pharmacies, supermarkets, etc.). This initiative raises many questions about clinical governance, clinician 'buy-in', and patient confidence/participation that have yet to be addressed. Indeed, the purported benefits versus risks have not substantively been assessed. Comprehensive needs analysis is warranted to assess what POCT is required in the community by clinicians and the public, as well as how best to meet those needs. Pathology and POCT staff are best positioned to assist in this process and should work closely and sensitively with their counterparts in the surrounding community - general practitioners, nurses, pharmacists, healthcare assistants to ensure a quality level consistent with hospital-based centralized laboratories.

\subsection{CONCLUSIONS}

POCT is not a replacement for conventional laboratory testing but rather a supplement to it. POCT results which are used for diagnosis or critical patient management decisions, or which give unexpected results should be confirmed by hospital laboratories to ensure accurate diagnosis and to facilitate correct patient management decisions.

The development and adoption of POC systems continues to increase, in part due to expanding test menus, lower costs and advances in data management infrastructure.

The POC market is growing due to advancements in technology and the demand for quick test results. In addition, the increase in the prevalence of infectious disease and lifestyle diseases like cardiac disease and diabetes along with the aging population, is increasing the demand for POC diagnostics. Awareness among people of the easy and convenient way to get faster test results is increasing the demand and physicians also prefer POC for their quick decision making when prescribing a disease-specific drug, which was previously unlikely when doctor started symptomatic treatment as the test procedure could take a long time. There is a tremendous demand for POC in the Americas region, and the increase in awareness in the Asia-Pacific region will lead to tremendous growth in the future as the highest population countries in Asia will drive it tremendously. The traditional method of clinical testing required skilled labour while POC required minimal training, thus reducing the costs of training and the time consumed to perform the test. The major restraint for POCT market is the unwillingness to change traditional treatment practice [17] [http://www.medgadget.com/2016/03/globalindustry-analysis-on-point-of-care-test-market-2016-2022.html].

Technological advances have made it possible to conduct many laboratory tests at, or near, the POC. These POCT devices give physicians rapid access to test results, allowing greater quality and efficiency in medical care [33]. The 
increased availability of POCT devices for more commonly performed routine tests would improve efficiency further. In the absence of any immediate health risk, physicians will probably wait to review all the results before reaching any patient management decision. Without universal POCT, prolonged turnarounds for laboratory transit and processing will continue to be the limiting stage in medical decision-making. As technological innovation provides more comprehensive POCT options for CBC, pregnancy testing, infectious disease, cancer screening, and other frequently ordered tests, nearpatient testing will become increasingly integrated into the traditional healthcare structure. The role and responsibilities of laboratory personnel will likely need reviewing and reworking as testing migrates away from the lab bench and closer to the bedside. Coordination with the central laboratory regarding quality assurance and regulatory matters will be crucial as technology allows the more efficient allocation of testing resources. Outside the hospital setting, POCT provides laboratory quality services to underserviced areas and general practitioners. Near-immediate test results allow patients and doctors to evaluate progress, review results, and establish treatment regimens in a single visit. This simple change can result in improved disease management, treatment adherence, and patient satisfaction. However, without the presence of an in-house laboratory, having measures in place to ensure adequate levels of quality assurance in POCT becomes critically important. As POCT continues to become an integral part of healthcare management, expansive quality assurance and training protocols should be established to ensure maximal benefits to patient care and efficiency in any setting.

The POCT has become an integral part of day today testing including home healthcare and by healthcare providers. The POCT is essential to provide the patient with quick and accurate treatment, thus avoiding the various side effects caused by prescribed drugs. The POC market is well established in the developed countries, which has the world's largest market share. However, developing countries such as those in the Asia-Pacific area have a huge potential due to the high population and increasing awareness of POC. The market is majorly driven by demand and awareness of the quick and easy results.

The success of a potential shift away from curative medicine, to predictive, personalized, and preemptive medicine could rely on the development of portable diagnostic and monitoring devices for POCT [34,35]. 


\subsubsection{Yesterday}

In the earliest days of medicine, healthcare was similar to POC in that it was delivered in the patient's home through physician house visits.

As medical discoveries were made and new technologies developed, care then shifted to specialized hospitals with an emphasis on curative medicine.

Large centralized laboratories were established, with cost-savings realized through the development of automated systems for analysing patient samples.

POC devices were used on a limited basis in the hospital for rapid analysis in intensive care units and for simple home testing, such as with pregnancy test kits.

\subsubsection{Today}

The emphasis of care is shifting toward the prevention and early detection of disease, as well as the management of multiple chronic conditions.

POCT gives immediate results in non-laboratory settings to support more patient-centred approaches to healthcare delivery.

The NIH supports the development of sensor, microsystem and low-cost imaging technologies for POCT. These instruments combine multiple analytical functions into self-contained, portable devices that can be used by nonspecialists to detect and diagnose disease, also enabling the selection of optimal therapies through patient screening and monitoring a patient's response to the chosen treatment.

Sensor technologies enable the rapid analysis of blood samples for several critical care assays, including blood chemistry, electrolytes, blood gases, and haematology.

Biosensors are used clinically for toxicology and drug screens, measurement of blood cells and blood coagulations, bedside diagnosis of heart disease through the detection of cardiac markers in the blood, and glucose self-testing.

Current developments in POCT are addressing the challenges of the diagnosis and treatment of cancer, stroke, and cardiac patients.

Circulating tumour cells (CTCs) that spread, or metastasize, from a primary malignant tumour to distant organs are responsible for $90 \%$ of cancer-related deaths, a number that exceeds 500,000 every year in the United States alone. The early detection of cancer might be possible through the capture and analysis of CTCs. In addition, the ability to capture and analyse CTCs in peripheral blood may be used in the development of therapeutic strategies that can be tailored to the individual patient and can monitor an individual's responses to cancer therapies.

Researchers supported by NIBIB have developed a unique microfluidic device capable of the efficient separation of CTCs from whole blood. This technology 
has wide implications both for advancing cancer biology research and for the clinical management of cancer, including detection, diagnosis, and monitoring.

\subsubsection{Tomorrow}

With the development of miniaturized devices and wireless communication, how doctors care for patients will change dramatically and the role patients take in their own healthcare will increase. Healthcare will become more personalized through tailoring interventions to individual patients.

The next decade will bring a new realm of precision and efficiency to the way information is transmitted and interpreted and thus the way medicine is practiced. In the future, clinicians may be able to improve the regulation of diet in infants with inborn metabolism issues through bedside monitoring. Currently, management of such diseases requires complex testing in a hospital setting. However, researchers are developing a chemical sensor, using a small sample of blood from a finger stick, which changes colour in response to metabolic irregularities. When such abnormalities are found, the diet of the infant can be adjusted immediately to prevent adverse effects such as mental retardation.

Low-cost diagnostic imaging devices can be used at the point-of-patient care for disadvantaged and under-served populations in the U.S. and in the developing world. The development of low-cost imaging devices could make affordable diagnostic imaging more widely available, particularly in remote or rural communities and small hospitals that do not have ready access to these technologies.

A new method using an optical probe for cervical cancer detection and treatment could significantly reduce the mortality rate worldwide. Combining a small optical imaging device with a treatment modality could provide both diagnosis and treatment of cervical cancer at the same time [36,37].

Several key challenges that must be addressed are bioanalytical performance, the miniaturization of microfluidic devices, material safety and disposal, changing CP specifications, data security and ownership, big data, and health economics feasibility.

\section{ACKNOWLEDGEMENT}

I would like to thank Mrs. Kristina Kumer and Mrs. Teja Fabjan for technical assistance in this work. 


\section{REFERENCES}

1. http://www.aacc.org/SiteCollectionDocuments/NACB/LMPG/POCT/POCT Entire LMPG.pdf (July 2016).

2. A.K. Yadav, N.N. Mohanty, M. Manu, K. Chand, S.K. Biswas, K.K. Rajak. Int. J. Livestock Res. 5(8) (2015) 9-17.

3. http://www.science.gov/topicpages/c/clonal+eosinophilia+point-ofcare.html (July 2016).

4. $\quad$ B. O'Farrell. "Evolution in Lateral Flow-Based Immunoassay Systems", Lateral Flow Immunoassay, Humana Press, USA, 2009.

5. The National Academy of Clinical Biochemistry. Laboratory Medicine Practice Guidelines Evidence-Based Practice For Point-Of-Care Testing. 2006

6. C.P. Price, A. St John, J.M. Hicks (Eds). Point-of-care testing, $2^{\text {nd }}$ Ed., AACC Press, Washington DC, 2004.

7. http://www.bountifulbraves.net/Comptech/Online\%20Information\%20Reso urces\%20Module/SearchingWorksheet1.pub (July 2016).

8. http://www.ohr.wisc.edu/weblisting/classified/PDDetailFormatted.aspx?vaci $\mathrm{d}=71042$ \&title $=41163$ (July 2016).

9. http://www.cdha.nshealth.ca/pathology-laboratory-medicine/clinicalservices/point-care-testing (July 2016).

10. http://www.ruh.nhs.uk/about/policies/documents/clinical_policies/blue_clin ical/Blue_772_Point_of_Care_Testing.pdf (July 2016).

11. C.P. Price, A. St John. Point-of-Care Testing for Managers and Policy makers: From Rapid Testing to Better Outcomes. AACC Press, 2006, p.p. 1-116.

12. http://www.diagnostics1.com/MANUAL/LFIA_Book\%5B1\%5D.pdf (July 2016).

13. http://bdx.com/vacutainer/labnotes/pdf/Volume8Number1.pdf (July 2016).

14. http://www.ncbi.nlm.nih.gov/pmc/articles/PMC4204237/(July 2016).

15. D. Huckle. Exp. Rev. .Med. Devices 3 (2006) 421-426.

16. J.L.V. Shaw. Practical Lab. Med. 4 (2016) 22-29.

17. Scientia Advisors. The point-of-care diagnostics market. Cambridge, MA, USA; 2013.

18. A. St John, C.P. Price. Clin. Biochem. Rev. 35(3) (2014) 155-167.

19. "Point of Care Diagnostic Testing World Market to Jump to $\$ 16.7$ Billion by 2018.", Marketwire Canada, April 132016 Issue (July 2016).

20. http://www.grandviewresearch.com/industry-analysis/point-of-care-pocdiagnostics-industry (July 2016).

21. https://www.trimarkpublications.com/products/Point-of-Care-DiagnosticTesting-World-Markets.html (July 2016) (July 2016).

22. C.P. Price, A. St John, L. Kricka. Point-of-Care Testing. Needs, opportunities and innovation, $3^{\text {rd }}$ Ed., Washington, USA, AACC Press, 2010.

23. Global Wearable Medical Device Market-Growth, Trends and Forecasts (2015-2020), Mordor Intelligence.

24. A. Larson, R. Greig-Pylypozuk, A. Huisman. Ups. J. Med. Sci. 120(1) (2015) $1-10$.

25. http://www.doh.wa.gov/Portals/1/Documents/2700/POCT.pdf (July 2016).

26. http://www.pointofcare.net/ (July 2016).

27. G. Abel. Exp. Rev. Mol. Diagn. (2015) 853-855.

28. http://www.doh.wa.gov/portals/1/Documents/2700/POCT.pdf (July 2016). 
29. http://lib.bioinfo.pl/pmid:208020 (July 2016).

30. O. Camacho-Ryan, R.L. Bertholf. Monitoring Point-of-Care Testing Compliance. AACC Publications. Clinical Laboratory News 2016;1:February.

31. J. Granz, P. Koerte, D. Stein. Point CareThe Journal of Near-Patient Testing and Technology 12 (2013) 76-79.

32. V.S. Kumar, P.B. Luppa, L.Y. Yeo, A. Ozcan, J.H.T. Luong. Trends Biotechnol. 33(11) (2015) 692-705.

33. A. Larsson, R. Greig-Pylypczuk, A. Huisman. Ups. J. Med. Sci. 120(1) (2015) $1-10$.

34. http://report.nih.gov/NIHfactsheets/ViewFactSheet.aspx?csid=112 (July 2016).

35. A. Travanty. Advance for Administrators of the Laboratory 20(11) (2011) 18-19.

36. http://www.medgadget.com/2016/03/global-industry-analysis-on-point-ofcare-test-market-2016-2022.html (July 2016).

37. J. DuBois. Point Care The Journal of Near-Patient Testing and Technology 9 (2001) 196-198. 